\title{
Trends in Biochemical Sciences
}

\section{CellPress \\ REVIEWS}

\section{Spotlight}

\section{Ki-67: More Hidden \\ behind a 'Classic \\ Proliferation Marker'}

\section{Raquel Sales Gil ${ }^{1}$ and Paola Vagnarelli $\mathbb{1}^{1, \star}$}

Nuclear antigen $\mathrm{Ki}-67$ is widely accepted as a cell proliferation marker in both research and cancer diagnostic settings. Despite its extensive use and clinical value, very little is still known about the biological function of Ki-67. A recent work published in Cell Reports has revealed important novel aspects of $\mathrm{Ki}-67$ regulation that could provide new and extended prognostic and therapeutic value.

Ki-67 was first identified in Hodgkin lymphoma cell nuclei as an antigen that is highly expressed in cycling cells but strongly downregulated in resting GOphase cells [1]. Since then, Ki-67 (also known as MKI67) is known to most of the scientific community as the prototype 'cell proliferation marker'. Its translational value is well established because it is widely used in the clinic for grading multiple types of cancers, with important prognostic value in large cancer cohorts $[2,3]$. In diagnostics, biopsies are analyzed and assigned a percent Ki-67-positive score; these Ki-67 scores are then used for prognosis and treatment (e.g., to assess potential benefits from chemotherapy in breast cancer). Despite its value in both laboratory and clinical settings, for several decades little has been known about the biological function of Ki-67. Therefore, understanding Ki-67 biology and regulation will provide essential knowledge that could potentially benefit the clinical diagnosis and treatment of cancer patients.
In the past few years several studies have revealed interesting aspects of Ki-67 biology, and Ki-67 is clearly much more interesting than merely being a marker of cell proliferation. Studies have uncovered that $\mathrm{Ki}-67$ is a protein Phosphatase 1 (PP1)interacting protein [4], with roles in the assembly of the perichromosomal layer on mitotic chromosomes [5]; it is also important for chromosome dispersal in mitosis [6], heterochromatin maintenance [7], and the cell cycle [7].

We spotlight here a recent article that has unveiled an important aspect of Ki-67 regulation [9] that provides added value with potential applications in the clinic.

In addition to the absence of this antigen in cells in GO phase, the levels and localization of Ki-67 are regulated according to the phase of the cell cycle. In interphase cells, Ki-67 is present in the nucleus, where it accumulates in the nucleoli in G2. At the onset of mitosis it relocalizes to the periphery of the mitotic chromosomes (the so-called perichromosomal layer), where it remains until the $\mathrm{G} 1$ nucleus reformation after chromosome segregation. After this, Ki-67 is reorganized first in small foci and then coalesces in the nucleoli (Figure 1). The overall levels of Ki-76 over the cell cycle are regulated by different converging mechanisms. One level of regulation is transcriptional: the promoter of the gene encoding $\mathrm{Ki}-67$ contains binding sites for the canonical G1-regulatory E2F family of transcription factors, leading Ki-67 mRNA levels to increase in G1 phase [8]. Regulation also takes place at the protein level: Ki-67 protein is degraded during $\mathrm{G} 1$ via the ubiquitin proteasome complex APC/CColh1, and this depends on inactivation of $\mathrm{Rb}$ by $\mathrm{CDK} 4 / 6[8,10]$. These two opposing mechanisms contribute to the final balance of this protein across the cell cycle, and explain the variable levels of the protein reported in literature.
In a recent study published in Cell Reports [9], Miller and colleagues used time-lapse microscopy and single-cell tracking of cell lines expressing a CDK2 activity sensor to quantify Ki-67 levels across the cell cycle. After cell division, the cells either immediately re-enter the cell cycle and increase CDK2 activity, or spontaneously turn off CDK2 and enter temporary quiescence; cells can then exit the quiescent state and re-enter the cell cycle by increasing their CDK2 activity. By tagging endogenous Ki-67 with a fluorescent protein using CRISPR-based genome editing, the authors were able to compare cells that rapidly re-entered the cell cycle with cells that had spent different amounts of time in GO phase before re-entering the cell cycle, as well as with cells that remained in G0 for a prolonged time. From these analyses the authors discovered that $\mathrm{Ki}$ 67 is uniformly degraded during the GO and G1 phases, but that its levels increase again several hours later, concurrently with the start of $S$ phase. After this point, Ki-67 levels rise steadily until mitotic exit. This characteristic seems to be a conserved trend across different cell lines.

Because Ki-67 levels fall as a function of the time the cell spends in quiescence, cells re-entering the cell cycle will have varying levels of Ki-67 depending on how long they have been quiescent (Figure 1). After a long period of quiescence, cells re-entering the cell cycle have no detectable Ki-67 signal in G1; this behavior well explains previous reports on the observed variability of Ki-67 staining. These observations therefore add the important concept that the level of Ki-67 expression represents a spectrum that is a function of both cellcycle phase and cell history.

With this knowledge, it is now possible to model Ki-67 protein decay in quiescence and predict the time elapsed since quiescent cells divided - simply by using Ki-67 levels as the readout. 


\section{Trends in Biochemical Sciences}

\section{CellPress \\ REVIEWS}

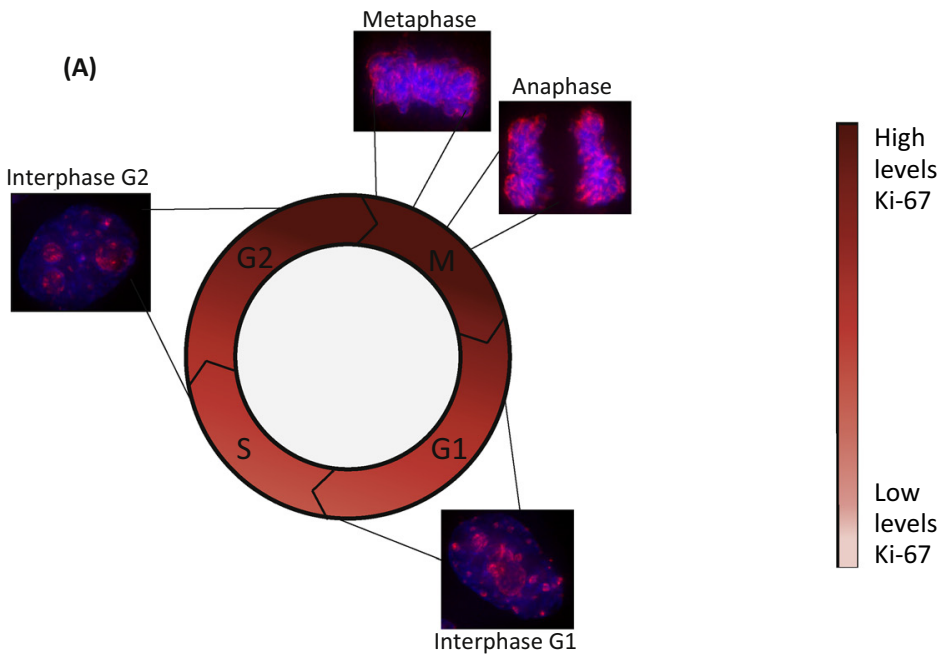

(B)

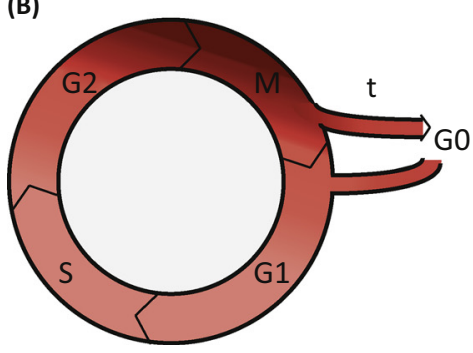

(C)

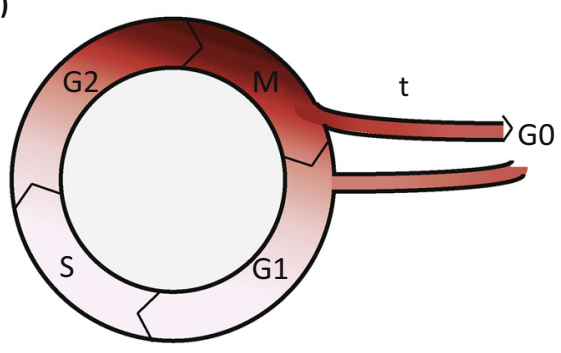

Trends in Biochemical Sciences

Figure 1. Ki-67 Gradients during the Cell Cycle. (A) Continuously dividing cells present maximum levels of Ki-67 during the G2/M-phase transition, and levels decrease gradually as the cell progresses through the cell cycle, being lowest at the G1/S-phase transition. Ki-67 localization also changes over the cell cycle. Images show Ki-67 staining in HeLa cells at different cell-cycle stages (Ki-67 in red, nuclei in blue). (B and C) In quiescence, Ki-67 levels also decrease gradually, thus when the quiescent cells re-enter the cycle, Ki-67 levels are decreased depending on the time the cells have spent in quiescence. Accordingly, upon re-entering the cell cycle, a cell that spends a short time in quiescence (B) will have more Ki-67 than a cell that has spent a long time in G0 (C). The red pattern represents Ki-67 levels, as shown in the color-scale key. Abbreviation: t, the time a cell spends in quiescence.

These findings have major implications for the clinic, and potentially could improve the diagnostic power of this already validated cancer marker. In fact, careful quantification of Ki-67 antibody staining can provide more information than simply whether a cell is proliferating or not; it can additionally distinguish a rapidly cycling cell (with a very short quiescence stage) from a slowly cycling cell that spends long periods in quiescence before re-entering the cell cycle. The predictive power of this analysis has been demonstrated by the authors using four different cell lines (MCF10A, 'normal' mammary epithelial cells; A375, skin malignant melanoma; OVCAR3, cisplatin-refractory ovarian carcinoma; and MCF7, breast adenocarcinoma) [9]; moreover, Ki-67 levels appear to be independent of the quiescence-inducing stimulus, served common role in the cell cycle.

Therefore, Ki-67 levels within a tumor might indicate how fast the tumor is growing. Rapidly growing tumors would have a distribution of Ki-67 levels (in G2-phase cells) concentrated towards the levels found in mitotic cells (high). By contrast, slowly growing G2 tumor cells would have possibly indicating that $\mathrm{Ki}-67$ plays a con-
Ki-67 levels more similar to the background Ki-67 levels found in adjacent non-cancerous tissue. This added classification could stratify tumors into more precise categories that can be then used for better prognostic and treatment purposes.

It would be of major interest now to join all these newly discovered biological aspects together, and identify the essential aspects that make this proliferation marker such a powerful prognostic tool and, perhaps in the future, a clinically relevant target.

\section{Acknowledgments}

Work in the laboratory of P.V. is supported by the Wellcome Trust and a Brunel Idea Award. P.V. is recipient of a Wellcome Trust Investigator Award in Science; R.S.G. is funded by an Isambard scholarship.

${ }^{1}$ College of Health and Life Science, Research Institute for Environment Health and Society, Brunel University London, London UB8 3PH, UK

\section{${ }^{*}$ Correspondence:}

Paola.Vagnarelli@Brunel.ac.uk (P. Vagnarelli).

https://doi.org/10.1016/j.tibs.2018.08.004

References

1. Gerdes, J. et al. (1983) Production of a mouse monoclonal antibody reactive with a human nuclear antigen associated with cell proliferation. Int. J. Cancer 31, 13-20

2. Pezzilli, R. et al. (2016) Ki-67 prognostic and therapeutic decision driven marker for pancreatic neuroendocrine neoplasms (PNENs): a systematic review. Adv. Med. Sci. 61, 147-153

3. Richards-Taylor, S. et al. (2016) The assessment of Ki-67 as a prognostic marker in neuroendocrine tumours: a systematic review and meta-analysis. J. Clin. Pathol. 69, 612-618

4. Kumar, G.S. et al. (2016) The Ki-67 and RepoMan mitotic phosphatases assemble via an identical, yet novel mechanism. Elife 5, e16539

5. Booth, D.G. et al. (2014) Ki-67 is a PP1-interacting protein that organises the mitotic chromosome periphery. Elife 3, e01641

6. Cuylen, S. et al. (2016) Ki-67 acts as a biological surfactant to disperse mitotic chromosomes. Nature 535, 308-312

7. Sobecki, M. et al. (2016) The cell proliferation antigen Ki-67 organises heterochromatin. Elife 5, e13722

8. Sobecki, M. et al. (2017) Cell-cycle regulation accounts for variability in Ki-67 expression levels. Cancer Res. 77, 2722-2734

9. Miller, I. et al. (2018) Ki67 is a graded rather than a binary marker of proliferation versus quiescence. Cell Rep. 24, 1105-1112

10. Chierico, L. et al. (2017) The role of the two splice variants and extranuclear pathway on Ki-67 regulation in non-cancer and cancer cells. PLoS One 12, e0171815 